\title{
Minimal sets of shifted values of the Euler totient function
}

\author{
Martin Kreh $^{1}$ and Katrin Neuenstein ${ }^{2}$ \\ ${ }^{1}$ Institute for Mathematics and Applied Computer Science, University of Hildesheim \\ Samelsonplatz 1, 31141 Hildesheim, Germany \\ e-mail: kreheimai.uni-hildesheim.de \\ ${ }^{2}$ Institute for Mathematics and Applied Computer Science, University of Hildesheim \\ Samelsonplatz 1, 31141 Hildesheim, Germany \\ e-mail: neuenste@uni-hildesheim.de
}

\begin{abstract}
In this article we determine the minimal set for some sets of natural numbers. The concept of minimal sets (in the context of natural numbers) appeared first in an article of Shallit, who determined, among others, the minimal set of the primes. By now, there are several articles about minimal sets. In this article we will expand results of Baoulina, Kreh and Steuding, who determined the minimal set of the sets $\varphi(\mathbb{N})$ and $\varphi(\mathbb{N})+3$. To this end, we will determine the minimal set of the sets $\varphi(\mathbb{N})+a$ for $1 \leq a \leq 5$.
\end{abstract}

Keywords: Minimal set, Euler totient function.

2010 Mathematics Subject Classification: 00A08, 11A25.

\section{Introduction}

In 2000, Shallit [10] introduced a problem concerning the decimal expansion of natural numbers. Given two natural numbers $x$ and $y$, we call $x$ a substring of $y$, written $x \triangleleft y$, if $x=y$ or if we can obtain $x$ from $y$ by deleting some of the digits of $y$. If $x$ is not a substring of $y$, we write $x \not y$. For example, we have $134 \triangleleft 918234 \triangleleft 98188293894$ but $123 \not 43021$. We call two natural numbers comparable, if $x \triangleleft y$ or $y \triangleleft x$, otherwise $x$ and $y$ are called incomparable. For a set $M \subset \mathbb{N}$ we 
want to find the smallest subset $A \subset M$ such that for all $m \in M$ there exists an $a \in A$ with $a \triangleleft m$. The set

$$
\mathcal{S}(M):=\{m \in M:\{n \in M: n<m, n \triangleleft m\}=\emptyset\}
$$

solves this problem, since any element of $M$ contains an element of $\mathcal{S}(M)$ as substring. The set $\mathcal{S}(M)$ is called minimal set of $M$ and its elements are called minimal. As a matter of fact, $\mathcal{S}(M)$ is finite for every $M \subset \mathbb{N}$. This is due to Higman's lemma, cf. [7, 11].

In [10], Shallit determined the minimal set of the primes and of the composite numbers. He showed

$$
\begin{aligned}
& \mathcal{S}(\mathbb{P})=\{2,3,5,7,11,19,41,61,89,409,449,499,881,991,6469,6949,9001 \\
&9049,9649,9949,60649,666649,946669,60000049,66000049,66600049\}
\end{aligned}
$$

and

$$
\begin{aligned}
\mathcal{S}(C)=\{4,6,8,9,10,12,15,20,21,22,25,27,30,32,33,35,50,51,52,55,57,70, & \\
& 72,75,77,111,117,171,371,711,713,731\}
\end{aligned}
$$

where $C$ denotes the composite numbers, i.e., $C=\mathbb{N} \backslash(\mathbb{P} \cup\{1\})$. Furthermore, he raised the conjecture, that

$$
\mathcal{S}\left(\left\{2^{n}: n \in \mathbb{N}_{0}\right\}\right)=\{1,2,4,8,65536\}
$$

and noted that this conjecture is true if any number $16^{m}$ with $m \geq 4$ contains at least one of the digits $1,2,4,8$. This conjecture is still unsolved. This shows that it can be difficult to determine the minimal set of a given set of natural numbers.

Recently, Bright, Devillers and Shallit [2] determined the minimal set of the primes in base $b$ for $2 \leq b \leq 30$ (for some of these bases under some conjectures). Gruber, Holzer and Kutrib $[4,5]$ have considered the problem of finding the minimal set from the viewpoint of theoretical computer science.

In [8], some more minimal sets have been determined, among them the minimal set of the set of natural numbers that can be written as a sum of two squares, as well as minimal sets of congruence classes. For congruence classes, an algorithm is developed that explicitly determines the minimal set. Furthermore, structural properties of minimal sets are examined.

In [6], the minimal set for the squarefree numbers has been determined. In [3], Baoulina, Kreh and Steuding determined some more minimal sets, among others, those of the set of natural numbers that can be written as a sum of three squares, as well as the minimal set for quadratic residues modulo $m$ and minimal sets of sets of values of arithmetic functions. The authors show, that

$$
\begin{aligned}
\mathcal{S}(\psi(\mathbb{N}))= & \{1,3,4,6,8,20,72,90,222,252,500,522,552,570,592,750,770, \\
& 992,7000, \underbrace{55 \ldots 5}_{69} 0\}
\end{aligned}
$$


and

$$
\mathcal{S}(\varphi(\mathbb{N}))=\{1,2,4,6,8,30,70,500,900,990,5590,9550,555555555550\},
$$

where $\psi$ is the Dedekind $\psi$-function,

$$
\psi(n):=\prod_{i=1}^{n}(p+1) p_{i}^{e_{i}-1} \quad \text { if } n=\prod_{i=1}^{n} p_{i}^{e_{i}},
$$

and $\varphi$ is the Euler totient function,

$$
\varphi(n):=\sum_{\substack{k=1 \\ \operatorname{gcd}(k, n)=1}}^{n} 1=\prod_{i=1}^{n}(p-1) p_{i}^{e_{i}-1} \quad \text { if } n=\prod_{i=1}^{n} p_{i}^{e_{i}} .
$$

The authors also state, without proof, the minimal set of the set $\varphi(\mathbb{N})+3:=\{\varphi(n)+3: n \in \mathbb{N}\}$.

In this article, we want to extend this result by considering the set

$$
\varphi(\mathbb{N})+a:=\{\varphi(n)+a: n \in \mathbb{N}\}
$$

for $a \in\{1,2,3,4,5\}$ and determining the respective minimal sets.

Here we restrict ourselves to the Euler totient function since it is one of the most important arithmetic functions. As will become clear later, some of the minimal sets can only be obtained subject to certain conjectures. These conjectures deal with questions of whether numbers of a certain form occur as the value of the Euler totient function. Heuristically, the more one-digit numbers are contained in $\varphi(\mathbb{N})+a$, the less numbers have to be considered (since all numbers that contain one of these digits cannot be contained in the minimal set). This is the reason why we deal only with the minimal sets for $a \leq 5$. For $a=6, \varphi(\mathbb{N})+6$ contains only two one-digit numbers. We hope that this article encourages others to develop methods to decide if numbers of a certain form can occur as the value of the Euler totient function.

We will use the following lemma, that has been proved as a part of a result in [3]:

Lemma 1.1 ([3, Theorem 4]). We have $555555555551 \in \mathbb{P}$, hence

$$
\varphi(555555555551)=555555555550
$$

For $l \leq 10$ we have $\underbrace{5 \ldots 5}_{\text {l times }} 0 \notin \varphi(\mathbb{N})$.

\section{Notation}

First we want to fix some notation. We consider natural numbers $\mathbb{N}=\{1,2, \ldots\}$ and their unique decimal representation, where each $n \in \mathbb{N}$ is given by $n=\sum_{j=0}^{k} \alpha_{j} 10^{j}$ for $k_{0} \in \mathbb{N}$ and a digit sequence $\alpha_{k} \alpha_{k-1} \ldots \alpha_{0}$ with $\alpha_{k} \neq 0$. Since this article is only concerned with the decimal representation, we will, by abuse of notation, call this digit sequence $n$. 
If $x$ and $y$ are two natural numbers, we denote with $x * y$ or $x y$ the natural number that is obtained by concatenation of the two digit sequences of $x$ and $y$. For two sets $M, L \subset \mathbb{N}$ we let

$$
M L:=M * L:=\{z \in \mathbb{N}: z=x * y, x \in M, y \in L\} .
$$

For $x, k \in \mathbb{N}$ we define inductively $x^{* k}:=x^{*(k-1)} * x, x^{* 1}:=x$ and let

$$
\{x\}^{*}=\left\{x^{* k}: k \in \mathbb{N}_{0}\right\} .
$$

Here $x^{* 0}$ means, that $x$ does not occur. This can be formalized with the empty word $\varepsilon$, for which we have $x * \varepsilon=\varepsilon * x=x$. For a set $M \subset\{0, \ldots, 9\}$ of digits we let

$$
M^{*}:=\left\{x \in \mathbb{N}_{0}:(d \triangleleft x, d \in\{0, \ldots, 9\}) \Rightarrow d \in M\right\} \cup \varepsilon .
$$

Hence $M^{*}$ contains (besides the empty word) all natural numbers that contain only the digits of $M$. For example, we have

$$
\{2\}\{1,3\}^{*}\{4\}\{5\}^{*}=\{24,214,234,245,2145,2345,21345,23145,2114,2334,2134, \ldots\} .
$$

\section{Results}

For some of the sets $\varphi(\mathbb{N})+a$ we can determine the minimal sets only by assuming certain conjectures. We have checked these conjectures for the first few cases (see also section 9), thus the conjectures seem reasonable to us.

Conjecture 1. Numbers of the form $n=69^{* k} 8$ with $k \in \mathbb{N}_{0}$ cannot occur as values of the Euler totient function.

Conjecture 2. Let $\alpha \in\{3,9\}^{*}$. Then, numbers of the form $\alpha 26$ and $\alpha 86$ cannot occur as values of the Euler totient function.

Conjecture 3. Numbers of the form $n=39^{* k} 8$ with $k \in \mathbb{N}_{0}$ cannot occur as values of the Euler totient function.

We show the following results:

Theorem 3.1. We have

$$
\mathcal{S}(\varphi(\mathbb{N})+1)=\{2,3,5,7,9,11,41,61,81\} .
$$

Theorem 3.2. If Conjecture 1 is correct, we have

$$
\mathcal{S}(\varphi(\mathbb{N})+2)=\{3,4,6,8,10,12,20,22,50,72,90,770,992,5592,9552,555555555552\} .
$$

If Conjecture 1 is wrong, we have

$$
\begin{aligned}
& \mathcal{S}(\varphi(\mathbb{N})+2)=\{3,4,6,8,10,12,20,22,50,72,90,770,992,5592,9552, \\
&\left.555555555552,70^{* m} 0\right\},
\end{aligned}
$$

where $m$ is the smallest natural number such that $69^{* m} 8 \in \varphi(\mathbb{N})$. 
Theorem 3.3. We have

$$
\mathcal{S}(\varphi(\mathbb{N})+3)=\{4,5,7,9,11,13,21,23,31,33,61,63,81,83\} .
$$

Theorem 3.4. If Conjecture 2 is correct, we have

$$
\begin{aligned}
& \mathcal{S}(\varphi(\mathbb{N})+4)=\{5,6,8,10,12,14,20,22,24,32,34,40,44,70,74,92, \\
&300,472,772,900,904,994\}
\end{aligned}
$$

If Conjecture 2 is wrong, $\mathcal{S}(\varphi(\mathbb{N})+4)$ contains the above values and in addition all minimal elements of the form $\alpha 30$ and $\alpha 90$ with $\alpha \in\{3,9\}^{*}$ such that $\alpha 26 \in \varphi(\mathbb{N})$, respectively, $\alpha 86 \in \varphi(\mathbb{N})$.

Theorem 3.5. If Conjecture 3 is correct, we have

$$
\begin{aligned}
& \mathcal{S}(\varphi(\mathbb{N})+5)=\{6,7,9,11,13,15,21,23,25,33,35,41,45,51,53,83,85,301,443,505, \\
&801,881,555555555555\} .
\end{aligned}
$$

If Conjecture 3 is wrong, we have

$$
\begin{aligned}
\mathcal{S}(\varphi(\mathbb{N})+5)=\{ & 6,7,9,11,13,15,21,23,25,33,35,41,45,51,53,83,85,301,443,505, \\
& \left.801,881,555555555555,40^{* m} 3\right\},
\end{aligned}
$$

where $m$ is the smallest natural number such that $39^{* m} 8 \in \varphi(\mathbb{N})$.

In the following five sections, we will prove the results for the respective minimal sets. We will finish with some remarks about the above conjectures.

To determine minimal sets, we first note that a subset of $M$ consisting of pairwise incomparable elements is not necessarily a subset of the minimal set of $M$ (for example, all two-digit numbers are incomparable, but none of them is contained in $\mathcal{S}(\mathbb{N})=\{1, \ldots, 9\}$ ). If, however, for any element $x$ in a set $A \subset M$ of pairwise incomparable elements there is no $y \in M, y \neq x$ such that $y \triangleleft x$, then $A$ is contained in the minimal set of $M$.

Thus, to determine the minimal sets, we first determine which digits lie in $M$ (these are also contained in $\mathcal{S}(M)$ ). Then, one checks, which elements with exactly $n$ digits (for increasing $n>1$ ) are contained in $M$ and cannot be truncated to some already obtained minimal element. This can be done by successively excluding certain combinations of digits. This process leads to the result, if one can successively exclude all possible combinations of digits. To this end, one needs to efficiently decide which elements are contained in $M$.

Hence for a number $m \in \mathbb{N}$ we need to decide whether $m$ can occur as a value of the Euler totient function or not. We will show this exemplarily for two numbers that appear in the proof for the sets $\varphi(\mathbb{N})+2$ and $\varphi(\mathbb{N})+5$, respectively. In the further course, the proof that certain numbers are not contained in $\varphi(\mathbb{N})$ is left to the reader.

We want to show that $5950 \notin \varphi(\mathbb{N})$ and $376 \notin \varphi(\mathbb{N})$. Since neither $5951=11 \cdot 541$ nor $377=13 \cdot 29$ is a prime, a potential $n$ with $\varphi(n)=5950$ or $\varphi(n)=376$ needs to be composite. 
Let $n=\prod_{i=1}^{k} p_{i}^{e_{i}}$. Assume that $k=1$, i.e., $n=p^{l}$ for a prime $p \neq 2$ and $l \geq 2$. Then, we get $(p-1) p^{l-1}=\varphi(n)=5950=2 \cdot 5^{2} \cdot 7 \cdot 17$. Thus we get $p \in\{5,7,17\}$, but none of the numbers $4,6,16$ is a divisor of 5950. For 376 we can argue analogously. Hence we have $k \geq 2$. Since the Euler totient function is multiplicative, we get $\varphi\left(p_{1}^{e_{1}}\right) \cdots \varphi\left(p_{k}^{e_{k}}\right)=5950=2 \cdot 5^{2} \cdot 7 \cdot 17$ in the first case. Since $\varphi\left(p_{i}^{e_{i}}\right)$ is even for every $i$, the left-hand side is divisible by 4 , but the right-hand side is not, hence we get $5950 \notin \varphi(\mathbb{N})$. For 376 we get $\varphi\left(p_{1}^{e_{1}}\right) \cdots \varphi\left(p_{k}^{e_{k}}\right)=376=2^{3} \cdot 47$. Hence we have $2 \leq k \leq 3$ and $94=2 \cdot 47$ or $188=2^{2} \cdot 47$ has to occur as a value of the Euler totient function. Again these numbers are not of the form $p-1$ for $p \in \mathbb{P}$ and we can show, exactly as we did above in the case $m=5950$, that a number $m$ with $\varphi(n)=94$ or $\varphi(n)=188$ cannot be composite. Thus we also have $376 \notin \varphi(\mathbb{N})$.

For concrete numbers $m$ we can show, using prime factorization, that $m$ cannot occur as a value of the Euler totient function. For general numbers of a certain form this can get difficult. This is why we need Conjectures 1, 2 and 3.

\section{The set $\mathcal{S}(\varphi(\mathbb{N})+1)$}

To begin with, all of the numbers mentioned in Theorem 3.1 are in $\varphi(\mathbb{N})+1$, since

$$
\begin{aligned}
\varphi(1)+1 & =2, & \varphi(3)+1 & =3, & \varphi(5)+1 & =5, \\
\varphi(7)+1 & =7, & \varphi(16)+1 & =9, & \varphi(11)+1 & =11, \\
\varphi(41)+1 & =41, & \varphi(61)+1 & =61, & \varphi(123)+1 & =81 .
\end{aligned}
$$

Furthermore, we have $1,4,6,8 \notin \varphi(\mathbb{N})+1$. Let $x \in \mathcal{S}(\varphi(\mathbb{N})+1)$ have at least two digits. Then, we have $x \in\{1,4,6,8\}\{0,1,4,6,8\}^{*}$ since otherwise $2,3,5,7,9 \triangleleft x$. Since $\varphi(n)$ is even for $n \geq 2, x$ is odd, hence its last digit is 1 . If $x$ contains another 1 , we have $11 \triangleleft x$ and $11 \in \mathcal{S}(\varphi(\mathbb{N})+1)$. If $x$ contains a 4,6 or an 8 , we can argue analogously. This covers all cases.

\section{The set $\mathcal{S}(\varphi(\mathbb{N})+2)$}

First we have

$$
\begin{aligned}
& \varphi(1)+2=3, \\
& \varphi(3)+2=4, \\
& \varphi(5)+2=6, \\
& \varphi(7)+2=8, \\
& \varphi(16)+2=10 \text {, } \\
& \varphi(11)+2=12 \text {, } \\
& \varphi(19)+2=20 \text {, } \\
& \varphi(25)+2=22, \\
& \varphi(65)+2=50 \text {, } \\
& \varphi(71)+2=72, \\
& \varphi(89)+2=90, \\
& \varphi(769)+2=770 \text {, } \\
& \varphi(991)+2=992 \text {, } \\
& \varphi(5591)+2=5592 \text {, } \\
& \varphi(9551)+2=9552
\end{aligned}
$$

and

$$
\varphi(555555555551)+2=5555555555552
$$

hence these elements are contained in $\varphi(\mathbb{N})+2$. Furthermore, we have $1,2,5,7,9 \notin \varphi(\mathbb{N})+2$. Now let $x \in \mathcal{S}(\varphi(\mathbb{N})+2)$ have at least two digits. Since $x$ is even, we get

$$
x \in\{1,2,5,7,9\}\{0,1,2,5,7,9\}^{*}\{0,2\} .
$$


We have $52,70,92 \notin \varphi(\mathbb{N})+2$, all other possible two-digit numbers are contained in $\mathcal{S}(\varphi(\mathbb{N})+2)$. So let $x$ have at least three digits. We consider two different cases.

1. The last digit of $x$ is 2 .

Then, $x$ is of the form $x=\alpha 2$ with $\alpha \in\{1,2,5,7,9\}\{0,1,2,5,7,9\}^{*}$. If $1,2,7 \triangleleft \alpha$, we have $12,22,72 \triangleleft x$ and $x$ cannot be contained in $\mathcal{S}(\varphi(\mathbb{N})+2)$. Hence let $\alpha \in\{5,9\}\{0,5,9\}^{*}$. Again, we distinguish two cases.

(a) The first digit of $x$ is 5 .

Then, $x$ is of the form $x=5 \beta 2$ with $\beta \in\{0,5,9\}^{*}$. If $0 \triangleleft \beta$, we have $50 \triangleleft x$, and if $99 \triangleleft \beta$, we have $992 \triangleleft x$. Hence $x$ cannot be contained in $\mathcal{S}(\varphi(\mathbb{N})+2)$ in these cases. If $\beta$ only contains the digit 5 , we have $555555555552 \triangleleft x$, since for $1 \leq k \leq 9$ we have $55^{* k} 2 \notin \varphi(\mathbb{N})+2$ due to Lemma 1.1. It remains to consider the cases where $\beta$ contains exactly one 9 and at most nine times the digit 5 . Since $5592 \in \mathcal{S}(\varphi(\mathbb{N})+2)$, we only need to consider the case $x=595^{* k} 2$ with $1 \leq k \leq 9$. For $k=1$ we have $x=5952$, which is not contained in $\varphi(\mathbb{N})+2$ (as shown above). For $k \geq 2$ we have $9552 \triangleleft x$, hence $x$ cannot be contained in $\mathcal{S}(\varphi(\mathbb{N})+2)$.

(b) The first digit of $x$ is 9 .

Then, $x$ is of the form $x=9 \beta 2$ with $\beta \in\{0,5,9\}^{*}$. If $0 \triangleleft \beta$, we have $90 \triangleleft x$, and if $9 \triangleleft \beta$, we have $992 \triangleleft x$ and $992 \in \mathcal{S}(\varphi(\mathbb{N})+2)$. So let us suppose that $\beta$ contains only the digit 5 . We have $952 \notin \varphi(\mathbb{N})+2$, and if $\beta$ contains at least two times the digit 5 , we have $9552 \triangleleft x$.

2. The last digit of $x$ is 0 .

Hence $x$ is of the form $x=\alpha 0$ with $\alpha \in\{1,2,5,7,9\}\{0,1,2,5,7,9\}^{*}$. If $1,2,5,9 \triangleleft \alpha$, we have $10,20,50,90 \triangleleft x$. It remains to consider the case $x=7 \beta 0$ with $\beta \in\{0,7\}^{*}$. If $7 \triangleleft \beta$, we have $770 \triangleleft x$. Thus it only remains to consider the case $x=70^{* k} 0$ with $k \in \mathbb{N}$, which is covered by Conjecture 1 .

\section{The set $\mathcal{S}(\varphi(\mathbb{N})+3)$}

Here we give the proof for the minimal set that is already mentioned in [3]. We have

$$
\begin{aligned}
& \varphi(2)+3=4, \quad \varphi(3)+3=5, \quad \varphi(5)+3=7, \quad \varphi(7)+3=9, \\
& \varphi(16)+3=11, \quad \varphi(11)+3=13, \quad \varphi(19)+3=21, \quad \varphi(25)+3=23, \\
& \varphi(58)+3=31, \quad \varphi(31)+3=33, \quad \varphi(59)+3=61, \quad \varphi(61)+3=63, \\
& \varphi(79)+3=81, \quad \varphi(123)+3=83
\end{aligned}
$$

and $1,2,3,6,8 \notin \varphi(\mathbb{N})+3$. If $x \in \mathcal{S}(\varphi(\mathbb{N})+3)$ has at least two digits, then $x$ is odd, hence its last digit is 1 or 3 . Then, we always have $y \triangleleft x$ for some $y \in\{11,13,21,23,31,33,61,63,81,83\}$. 


\section{$7 \quad$ The set $\mathcal{S}(\varphi(\mathbb{N})+4)$}

First we have

$$
\begin{aligned}
& \varphi(1)+4=5, \quad \varphi(3)+4=6, \quad \varphi(5)+4=8, \quad \varphi(7)+4=10, \\
& \varphi(16)+4=12, \quad \varphi(11)+4=14, \quad \varphi(17)+4=20, \quad \varphi(19)+4=22, \\
& \varphi(25)+4=24, \quad \varphi(29)+4=32, \quad \varphi(31)+4=34, \quad \varphi(37)+4=40, \\
& \varphi(41)+4=44, \quad \varphi(67)+4=70, \quad \varphi(71)+4=74, \quad \varphi(89)+4=92, \\
& \varphi(447)+4=300, \quad \varphi(553)+4=472, \quad \varphi(769)+4=772, \quad \varphi(1347)+4=900, \\
& \varphi(1057)+4=904, \quad \varphi(991)+4=994 \text {. }
\end{aligned}
$$

Further we have $1,2,3,4,7,9 \notin \varphi(\mathbb{N})+4$. If $x \in \mathcal{S}(\varphi(\mathbb{N})+4)$ has at least two digits, we get, since $x$ is even, that $x \in\{1,2,3,4,7,9\}\{0,1,2,3,4,7,9\}^{*}\{0,2,4\}$. We have $30,42,72,90,94 \notin$ $\varphi(\mathbb{N})+4$, the other possible values for $x$ are contained in $\varphi(\mathbb{N})+4$, and hence they are also contained in $\mathcal{S}(\varphi(\mathbb{N})+4)$. Now let $x$ have at least three digits. We consider three cases.

1. The last digit of $x$ is 2 .

Hence $x=\alpha 2$ with $\alpha \in\{1,2,3,4,7,9\}\{0,1,2,3,4,7,9\}^{*}$. If $1,2,3,9 \triangleleft \alpha$, then we have $12,22,32,92 \triangleleft x$ and thus $x \notin \mathcal{S}(\varphi(\mathbb{N})+4)$. Now assume that $\alpha \in\{4,7\}\{0,4,7\}^{*}$.

(a) Suppose that the first digit of $x$ is 7 , i.e., $x=7 \beta 2$ with $\beta \in\{0,4,7\}^{*}$. If $0,4,7 \triangleleft \beta$, we get $70,74,772 \triangleleft x$ and this covers all cases.

(b) Suppose that the first digit of $x$ is 4 , i.e., $x=4 \beta 2$ with $\beta \in\{0,4,7\}^{*}$. If $0,4,7 \triangleleft \beta$, we get $40,44,472 \triangleleft x$ and this covers all cases.

2. The last digit of $x$ is 4 .

Hence we have $x=\alpha 4$ with $\alpha \in\{1,2,3,4,7,9\}\{0,1,2,3,4,7,9\}^{*}$. If $1,2,3,4,7 \triangleleft \alpha$, we get $14,24,34,44,74 \triangleleft x$. So suppose that the first digit of $x$ is 9 , i.e., $x=9 \beta 4$ with $\beta \in\{0,9\}^{*}$. If $0,9 \triangleleft \beta$, we have $904,994 \triangleleft x$ and this covers all cases.

3. The last digit of $x$ is 0 .

Hence we have $x=\alpha 0$ with $\alpha \in\{1,2,3,4,7,9\}\{0,1,2,3,4,7,9\}^{*}$. If $1,2,4,7 \triangleleft \alpha$, we get $10,20,40,70 \triangleleft x$. Now let $\alpha \in\{3,9\}\{0,3,9\}^{*}$. If $0 \triangleleft \alpha$, we have $300 \triangleleft x$ or $900 \triangleleft x$. It remains to consider the case $x=\alpha 0$ with $\alpha \in\{3,9\}^{*}$. This is covered by Conjecture 2 . 


\section{The set $\mathcal{S}(\varphi(\mathbb{N})+5)$}

We have

$$
\begin{aligned}
& \varphi(1)+5=6, \quad \varphi(3)+5=7, \quad \varphi(5)+5=9, \quad \varphi(7)+5=11, \\
& \varphi(16)+5=13, \quad \varphi(11)+5=15, \quad \varphi(17)+5=21, \quad \varphi(19)+5=23, \\
& \varphi(25)+5=25, \quad \varphi(29)+5=33, \quad \varphi(31)+5=35, \quad \varphi(37)+5=41, \\
& \varphi(41)+5=45, \quad \varphi(47)+5=51, \quad \varphi(65)+5=53, \quad \varphi(79)+5=83, \\
& \varphi(123)+5=85, \quad \varphi(447)+5=301, \quad \varphi(439)+5=443, \quad \varphi(625)+5=505 \text {, } \\
& \varphi(797)+5=801, \quad \varphi(877)+5=881
\end{aligned}
$$

and

$$
\varphi(555555555551)+5=555555555555 .
$$

We further have $1,2,3,4,5,8 \notin \varphi(\mathbb{N})+5$. Let $x \in \mathcal{S}(\varphi(\mathbb{N})+5)$ have at least two digits. Since $x$ is odd, we have $x \in\{1,2,3,4,5,8\}\{0,1,2,3,4,5,8\}^{*}\{1,3,5\}$. We have $31,43,55,81 \notin$ $\varphi(\mathbb{N})+5$, all other possible values of $x$ are contained in $\mathcal{S}(\varphi(\mathbb{N})+5)$. Now let $x$ have at least three digits. We consider three cases.

1. The last digit of $x$ is 1 .

Then, $x=\alpha 1$ with $\alpha \in\{1,2,3,4,5,8\}\{0,1,2,3,4,5,8\}^{*}$. If $1,2,4,5 \triangleleft \alpha$, we have $11,21,41,51 \triangleleft x$. Now let $\alpha \in\{3,8\}\{0,3,8\}^{*}$.

(a) Suppose that the first digit of $x$ is 3, i.e., $x=3 \beta 1$ with $\beta \in\{0,3,8\}$. If $3,0,88 \triangleleft \beta$, we have $33,301,881 \triangleleft x$. It remains to consider the case $x=381$, but we have $381 \notin \varphi(\mathbb{N})+5$.

(b) Suppose that the first digit of $x$ is 8 , i.e., $x=8 \beta 1$ with $\beta \in\{0,3,8\}$. If $3,0,8 \triangleleft \beta$, we have $83,801,881 \triangleleft x$ and this covers all cases.

2. The last digit of $x$ is 5 .

Hence $x=\alpha 5$ with $\alpha \in\{1,2,3,4,5,8\}\{0,1,2,3,4,5,8\}^{*}$. If $1,2,3,4,8 \triangleleft \alpha$, we have $15,25,35,45,85 \triangleleft x$. Now let $\alpha \in\{5\}\{0,5\}^{*}$. If $0 \triangleleft \alpha$, we have $505 \triangleleft x$. It remains to consider the case $x=5^{* k}$. Due to Lemma 1.1, we get that 555555555555 is the first such natural number that is contained in $\varphi(\mathbb{N})+5$.

3. The last digit of $x$ is 3 .

Then, $x=\alpha 3$ with $\alpha \in\{1,2,3,4,5,8\}\{0,1,2,3,4,5,8\}^{*}$. If $1,2,3,5,8 \triangleleft \alpha$, we have $13,23,33,53,83 \triangleleft x$. Let $\alpha \in\{4\}\{0,4\}^{*}$. If $4 \triangleleft \alpha$, we have $443 \triangleleft x$. In the other case, we have $x=40^{* k} 3$, which is covered by Conjecture 3 . 


\section{Remarks about the conjectures}

In this section we will give some remarks and possible approaches on the conjectures. There are similar conjectures in related work on minimal sets, see, for example, $[8,2]$. These conjectures involve only sets $A^{*}$ with $|A|=1$ (as in Conjectures 1 and 3). Since Conjecture 2 involves a set $A^{*}$ with $|A|=2$, this seems to be more inaccesible (since there are more numbers to check) and also (at least heuristically) more unlikely to hold.

Similar to Shallit's conjecture about the powers of 2 , it seems to be difficult to prove Conjectures 1,2 and 3. Let us denote the number $69^{* l} 8$ by $\alpha_{l}$ and the number $39^{* l} 8$ by $\beta_{l}$. Since $\alpha_{l}+1 \equiv \beta_{l}+1 \equiv 0 \bmod 3$, these numbers are not of the form $p-1$ for a prime $p$. Since further $\alpha_{l} \equiv \beta_{l} \equiv 2 \bmod 4$, a potential $m$ with $\varphi(m)=\alpha_{l}$ or $\varphi(m)=\beta_{l}$ has to be of the form $m=p^{k}$ or $m=2 p^{k}$ with a prime $p \equiv 3 \bmod 4$ and $k \geq 2$, since any other prime factor and any other power of 2 would imply the divisibility of $\varphi(m)$ by 4 . Since $\varphi\left(p^{k}\right)=\varphi\left(2 p^{k}\right)$, we can restrict ourselves to the case $\alpha_{l}=\varphi\left(p^{k}\right)=p^{k-1}(p-1)$, respectively, $\beta_{l}=\varphi\left(p^{k}\right)=p^{k-1}(p-1)$. We can further deduce that $k \geq 3$ : If $k=2$, we are looking for primes that satisfy the equation $x^{2}-x-\alpha_{l}=0$. Using the formula for quadratic equations we get as solutions $\frac{1}{2} \pm \frac{\sqrt{4 \alpha_{l}+1}}{2}$. This can only be an integer if $4 \alpha_{l}+1$ is a square. Since $4 \alpha_{l}+1 \equiv 3 \bmod 10$, this is not possible. The same argument for $\beta_{l}$ shows that we necessarily have $k \geq 3$.

Computer calculations show that for $1 \leq l \leq 100$ neither $\alpha_{l}$ nor $\beta_{l}$ are of this form. In fact, in these 200 cases there are only 4 cases in which we have a prime factor $p$ in the factorization of $\alpha_{l}$ or $\beta_{l}$ with an exponent greater than 1 . In all these cases, the product of the remaining prime factors is far greater than $p-1$.

One can also use further congruence considerations to get more restrictions on a potential prime $p$ with $\varphi\left(p^{k}\right)=\alpha_{l}$ or $\varphi\left(p^{k}\right)=\beta_{l}$. We show this exemplarily for the case $\varphi\left(p^{k}\right)=\alpha_{l}$.

We have $\alpha_{l} \equiv 2 \bmod 3$. Since $\alpha_{l}=p^{k-1}(p-1)$, we get $p-1 \not \equiv 0 \bmod 3$. This implies $p \equiv 2 \bmod 3$. We also get $k \equiv 0 \bmod 2$, since otherwise $p^{k-1}(p-1) \equiv 1 \bmod 3$. Since $\alpha_{l} \equiv 3 \bmod 5$ and $k$ needs to be even, we further get $p \equiv 2 \bmod 5$ and $k \equiv 0 \bmod 4$. Moreover, $\alpha_{l} \equiv 5 \bmod 9$ implies either $p \equiv 2 \bmod 9$ and $k \equiv 0 \bmod 6$ or $p \equiv 5 \bmod 9$ and $k \equiv 4 \bmod 6$.

Combining these congruences with the Chinese Remainder Theorem, we get that either $p \equiv$ $2 \bmod 45$ and $k \equiv 0 \bmod 12$, or $p \equiv 32 \bmod 45$ and $l \equiv 4 \bmod 12$.

If $l$ is big enough, we can get further congruence conditions modulo powers of 2 . For example, if $l \geq 2$, we have $\alpha_{l} \equiv 6 \bmod 8$, which implies $p \equiv 3 \bmod 8$. If $l \geq 3$, an analogous argument yields $p \equiv 11 \bmod 16$.

Starting from $p \equiv a \bmod 2^{m}$, we get $p \equiv a \bmod 2^{m+1}$ or $p \equiv a+2^{m} \bmod 2^{m+1}$. Which of these cases occurs can be determined with Hensel's Lemma: If we consider the polynomial $f(x)=x^{k}-x^{k-1}-\alpha_{l}$, we get $f(a) \equiv f(p)=p^{k}-p^{k-1}-\alpha_{l}=0 \bmod 2^{m}$ and $f^{\prime}(a)=k a^{k-2}(a-$ 1) $+a^{k-2} \equiv 1 \bmod 2$. Thus, there exists a unique $s$ modulo $2^{m+1}$ such that $f(s) \equiv 0 \bmod 2^{m+1}$ and $s \equiv a \bmod 2^{m}$. This is given by $s=a+f(a)$. But since $k$ is not fixed, different possibilities can occur if $k$ varies.

If one could give congruence conditions on $p$ and $k$ for general powers of 2 , one could try to use the congruences $p \equiv a \bmod m$ and $k \equiv b \bmod n$ to conclude that $p^{k-1}>\alpha_{l}$, which would yield the impossibility of the representation $\alpha_{l}=p^{k-1}(p-1)$. Using the congruence conditions 
developed above, this works for $l$ small enough: If $l \geq 3$, we get $p \geq 587$ and $k \geq 12$ or $p \geq 347$ and $k \geq 4$, and this yields $p^{k-1}>\alpha_{l}$ for $l \leq 8$.

To show the conjecture for $\alpha_{l}$, one could also consider the Diophantine exponential equation $7 \cdot 10^{l}=p^{k}-p^{k-1}+2$, since $\alpha_{l-1}=7 \cdot 10^{l}-2$. Equations of this type have, for example, been considered by Baker. In [1], he develops upper bounds on $p$, but these bounds are increasing when $l$ increases (besides, these bounds are so huge that the remaining cases cannot be checked with computer calculations). Maybe a consideration of the particular equation above with similar methods can yield a solution to Conjecture 1 .

In Conjecture 2 we have to consider much more natural numbers for a given number of digits. Here computer calculations show that the conjecture holds for all such numbers with at most 20 digits.

Finally, note that Maier and Pomerance [9] showed that the number of distinct values of the Euler totient function not exceeding $x$ is equal to

$$
\frac{x}{\log x} \exp \left((C+o(1))(\log \log \log x)^{2}\right)
$$

for a positive constant $C$.

Hence, the probability that a 'random' number not exceeding $x$ is in the image of the totient function is roughly $\frac{1}{\log x}$. So if $x$ has $k$ digits, the probability that $x$ is in the image of the totient function is roughly $\frac{1}{k}$. If we suppose that the numbers considered in the conjectures are 'random', this means that large counterexamples get more unlikely, but since the harmonic series diverges, this would predict infinitely many counterexamples (and even small counterexamples for Conjecture 2).

On the other hand, as shown above, counterexamples to Conjectures 1 and 3 have to be of the form $p^{k}-p^{k-1}$ with $p, k \geq 3$. Note that each number of this form is the value of the totient function of a prime power $p^{k}$ with $p, k \geq 3$ and if $p^{k}-p^{k-1} \leq x$, we have $p^{k} \leq \frac{p}{p-1} x<2 x$. Hence the number of integers not exceeding $x$ of this form is less than the number of prime powers $p^{k}$ not exceeding $2 x$ with $p, k \geq 3$. If we denote this number by $\Pi_{3}(2 x)$, we have

$$
\Pi_{3}(2 x)=\sum_{k=3}^{\left\lfloor\log _{3}(2 x)\right\rfloor} \pi\left((2 x)^{\frac{1}{k}}\right),
$$

where $\pi(x)$ denotes the number of primes not exceeding $x$ and $\lfloor x\rfloor$ denotes the greatest integer less than or equal to $x$. Using the prime number theorem, we get as a rough estimate

$$
\begin{aligned}
\Pi_{3}(2 x) & =\mathcal{O}\left(\sum_{k=3}^{\left\lfloor\log _{3}(2 x)\right\rfloor} \frac{(2 x)^{\frac{1}{k}}}{\frac{1}{k} \log 2 x}\right) \\
& =\mathcal{O}\left(\frac{x^{\frac{1}{3}}}{\log x} \sum_{k=3}^{\left\lfloor\log _{3}(2 x)\right\rfloor} k\right) \\
& =\mathcal{O}\left(\frac{x^{\frac{1}{3}}}{\log x}\left(\log _{3}(2 x)\right)^{2}\right)=\mathcal{O}\left(x^{\frac{1}{3}} \log x\right) .
\end{aligned}
$$


This implies, that the probability, that a 'random' $k$-digit number is of the above form, is at most $\mathcal{O}\left(k \cdot 10^{-\frac{2 k}{3}}\right)$. Hence, if $\alpha_{l}$ and $\beta_{l}$ are 'random' numbers, at most finitely many counterexamples to Conjectures 1 and 3 exist.

\section{Acknowledgements}

We thank the anonymous reviewers for their helpful comments.

\section{References}

[1] Baker, A. (1969). Bounds for the solutions of the hyperelliptic equation, Proc. Camb. Phil. Soc., 65, 439-444.

[2] Bright, C., Devillers, R., \& Shallit, J. (2016). Minimal elements for the prime numbers, Exp. Math., 25(3), 321-331.

[3] Baoulina, I. N., Kreh, M., \& Steuding, J. (2017). Deleting digits, Math. Gaz., 101 (550), $60-68$.

[4] Gruber, H., Holzer, M., \& Kutrib, M. (2007). The size of Higman-Haines sets, Theoret. Comput. Sci., 387 (2), 167-176.

[5] Gruber, H., Holzer, M., \& Kutrib, M. (2009). More on the size of Higman-Haines sets: effective constructions, Fund. Inform., 91 (1), 105-121.

[6] Goldschmied, M. (2013). Über Mengen natürlicher Zahlen und ihre Ziffernfolgen, Bachelorthesis, Available online at: https: / /www. mathematik.uni-wuerzburg.de/ steuding/goldsch.pdf.

[7] Higman, G. (1952). Ordering by divisibility in abstract algebras, Proc. London Math. Soc., $2,326-336$.

[8] Kreh, M. (2015). Minimal sets, J. Integer Seq., 18 (5), Article 15.5.3, 38 pp.

[9] Maier, H. \& Pomerance, C. (1988). On the number of distinct values of Euler's $\varphi$-function, Acta Arith., 49 (3), 263-275

[10] Shallit, J. (2000). Minimal primes, J. Recreat. Math., 30, 113-117.

[11] Sakarovitch J. \& and Simon, I. (1983). Subwords, in: Combinatorics on Words, Encyclopedia of Mathematics and Its Applications, Vol. 17, Addison-Wesley. 